\title{
Analysis of the Planar Boundary Layer of Ionized Gas Adjacent to Porous Contour
}

\author{
S. Savić, B. Obrović, I. A. Soloviev, V. Nikolić
}

\begin{abstract}
Planar ionized gas (air) flow in the boundary layer under the conditions of the socalled equilibrium ionization is studied in this paper. The contour of the body within the fluid is porous. The ionized gas flows through the magnetic field of the power $B_{m}=B_{m}(x) \dot{\mathrm{I}}$ is assumed that the ionized gas electro-conductivity is a function of the ratio of the longitudinal velocity and the velocity at the outer edge of the boundary layer. Using the general similarity method, the governing boundary layer equations are brought to a generalized form, and as such solved numerically. Some conclusions concerning behavior of the boundary layer physical quantities and characteristics of compressible fluid flow have been drawn.
\end{abstract}

Keywords: boundary layer, ionized gas, electro-conductivity, porous contour, numerical solution.

\section{Introduction}

The paper investigates the ionized gas flow in the boundary layer adjacent to the body of porous contour. The boundary layer is studied under conditions of equilibrium ionization, where the ionized gas flow is planar.

The primary objective of this study is to obtain generalized boundary layer equations using the generalized similarity method and to solve the obtained equations.

Due to high temperatures at supersonic gas flow, both gas dissociation and ionization occur and the ionized gas becomes electro-conductive. When the ionized gas flows in the magnetic field of the power $\vec{B}_{m}$, an electric flow occurs. The most important results in dissociated gas flow investigation are presented in the [1]. Authors [2-4] studied the dissociated gas flow in the boundary layer and achieved significant results.

Manuscript received May 16, 2013; accepted August 19, 2013.

S. Savić, B. Obrović are with the Faculty of Engineering, University of Kragujevac, Serbia; V. Nikolić, is with the State University of Novi Pazar, Novi Pazar, Serbia; I. A. Soloviev is with the State University of Land Tenure, 15 Kazakova Street, Moscow 105064, Russia 
The paper [2] gives a detailed investigation of the boundary layer ionized gas flow along a planar nonporous plate when a magnetic field is present. The objective of that investigation was to obtain auto-model solution. Distribution of the power of the outer magnetic field is defined in order to bring the governing partial boundary layer equations down to simple differential equations.

This paper investigates the ionized gas flow in the boundary layer adjacent to the body of an arbitrary shape. The flow is planar and steady and the contour of the body within the fluid is porous. The outer magnetic field is perpendicular to the contour of the body. Since the thickness of the boundary layer is small, the power of the magnetic field is $B_{m y}=$ $B_{m}=B_{m}(x)$ and the magnetic Reynolds number is small. With the velocity $v_{w}(x)$, the gas is injected i.e., ejected perpendicularly to the porous wall of the body within the fluid.

\section{Governing equations}

The equations of the laminar steady and planar boundary layer under conditions of equilibrium ionization [2] are:

$$
\begin{aligned}
& \frac{\partial}{\partial x}(\rho u)+\frac{\partial}{\partial y}(\rho v)=0 \\
& \rho u \frac{\partial u}{\partial x}+\rho v \frac{\partial u}{\partial y}=-\frac{d p}{d x}+\frac{\partial}{\partial y}\left(\mu \frac{\partial u}{\partial y}\right)-\sigma B_{m}^{2} u \\
& \rho u \frac{\partial h}{\partial x}+\rho v \frac{\partial h}{\partial y}=u \frac{d p}{d x}+\mu\left(\frac{\partial u}{\partial y}\right)^{2}+\frac{\partial}{\partial y}\left(\frac{\mu}{\operatorname{Pr}} \frac{\partial h}{\partial y}\right)+\sigma B_{m}^{2} u^{2}
\end{aligned}
$$

The corresponding boundary conditions are:

$$
\begin{aligned}
& u=0, \quad v=v_{w}(x), \quad h=h_{w} \quad \text { for } y=0, \\
& u \rightarrow u_{e}(x), \quad h \rightarrow h_{e}(x) \quad \text { for } y \rightarrow \infty .
\end{aligned}
$$

The equation system (1) represents a mathematical model of this flow problem. The equations of the governing system are: the continuity equation, the dynamic equation, and the energy equation of the ionized gas boundary layer, respectively. In the equation system (1) and in the boundary conditions (2), the notation common in the boundary layer theory is used for the physical values and the indices. Here, $u(x, y)$ is the longitudinal projection of velocity in the boundary layer, $v(x, y)$ - transversal projection, $\rho$ - ionized gas density, $p$ - pressure, $h$ - enthalpy, $\mu$ - coefficient of dynamic viscosity, $\sigma$ - ionized gas electroconductivity and $\mathrm{Pr}$ - Prandtl number. The indices stand for: $w$-values on the wall of the body within the fluid and $e-$ physical values at the outer edge of the boundary layer.

Electro-conductivity $\sigma$ is an important physical parameter of the ionized gas. In general, it is a variable that depends on the temperature [2], i.e. the gas enthalpy $h$. Since we 
do not know the exact law on variation of electro-conductivity, by analogy with MHD boundary layer $[5,6]$ it is assumed that the electro-conductivity is a function of the ratio of the longitudinal velocity and the velocity at the outer edge of the boundary layer

$$
\sigma=\sigma_{0}\left(1-\frac{u}{u_{e}}\right), \quad\left(\sigma_{0}=\text { const. }\right) .
$$

Based on the boundary conditions for the velocity and density at the outer edge of the boundary layer, the pressure $p(x)$ can be eliminated from the system (1). This way, the governing equation system (1) is brought down to:

$$
\begin{aligned}
& \frac{\partial}{\partial x}(\rho u)+\frac{\partial}{\partial y}(\rho v)=0 \\
& \rho u \frac{\partial u}{\partial x}+\rho v \frac{\partial u}{\partial y}=\rho_{e} u_{e} \frac{d u_{e}}{d x}+\frac{\partial}{\partial y}\left(\mu \frac{\partial u}{\partial y}\right)-\sigma_{0} B_{m}^{2} u\left(1-\frac{u}{u_{e}}\right) \\
& \rho u \frac{\partial h}{\partial x}+\rho v \frac{\partial h}{\partial y}=-u \rho_{e} u_{e} \frac{d u_{e}}{d x}+\mu\left(\frac{\partial u}{\partial y}\right)^{2}+\frac{\partial}{\partial y}\left(\frac{\mu}{\operatorname{Pr}} \frac{\partial h}{\partial y}\right)+\sigma_{0} B_{m}^{2} u^{2}\left(1-\frac{u}{u_{e}}\right)
\end{aligned}
$$

where the boundary conditions (2) remain unchanged.

\section{Transformation of the equations}

Instead of the physical coordinates $x, y$, new variables are introduced in the form of

$$
s(x)=\frac{1}{\rho_{0} \mu_{0}} \int_{0}^{x} \rho_{w} \mu_{w} d x, \quad z(x, y)=\frac{1}{\rho_{0}} \int_{0}^{y} \rho d y .
$$

The stream function $\psi(s, z)$ is introduced in accordance with the expressions

$$
u=\frac{\partial \psi}{\partial z}, \tilde{v}=\frac{\rho_{0} \mu_{0}}{\rho_{w} \mu_{w}}\left(u \frac{\partial z}{\partial x}+v \frac{\rho}{\rho_{0}}\right)=-\frac{\partial \psi}{\partial s},
$$

that are based on the continuity equation.

In transformations (5) and (6) and further on, the values $\rho_{0}$ and $\mu_{0}=\rho_{0} v_{0}$ stand for the known values of the density and dynamic viscosity of the ionized gas (air), while $v_{0}$ represents the kinematical viscosity at a concrete point of the boundary layer. Here, $\rho_{w}$ and $\mu_{w}$ denote the given distributions of these values on the wall of the body within the fluid.

In order to apply the general similarity method, the corresponding momentum equation is obtained from the first two equations of the system (4) by integration transversally to the boundary layer (from the inner to the outer edge of the boundary layer) and by transformation of the variables. The momentum equation can take the three following forms:

$$
\frac{d Z^{* *}}{d s}=\frac{F_{m p}}{u_{e}}, \quad \frac{d f}{d s}=\frac{u_{e}^{\prime}}{u_{e}} F_{m p}+\frac{u_{e}^{\prime \prime}}{u_{e}^{\prime}} f, \quad \frac{1}{\Delta^{* *}} \frac{d \Delta^{* *}}{d s}=\frac{u_{e}^{\prime}}{u_{e}} \frac{F_{m p}}{2 f} ;
$$


where prim stands for the derivative per the variable $s$.

In order to obtain the momentum equation, we introduce the parameter of the form $f(s)$, magnetic parameter $g(s)$, conditional displacement thickness $\Delta^{*}$, conditional momentum loss thickness $\Delta^{* *}$, conditional thickness $\Delta_{1}^{*}$, shear stress on the wall of the body within the fluid $\tau_{w}$, non-dimensional friction function $\zeta(s)$ and non-dimensional values $H$ and $H_{1}$. These values are defined as:

$$
\begin{aligned}
& Z^{* *}=\frac{\Delta^{2}}{v_{0}}, \quad f(s)=\frac{u_{e}^{\prime} \Delta^{* *^{2}}}{v_{0}}=u_{e}^{\prime} Z^{* *}=f_{1}(s), \quad g(s)=N_{\sigma} Z^{* *}=g_{1}(s), \\
& \Delta^{*}(s)=\int_{0}^{\infty}\left(\frac{\rho_{e}}{\rho}-\frac{u}{u_{e}}\right) d z, \quad \Delta^{* *}(s)=\int_{0}^{\infty} \frac{u}{u_{e}}\left(1-\frac{u}{u_{e}}\right) d z \\
& \Delta_{1}^{* *}(s)=\int_{0}^{\infty} \frac{u}{u_{e}}\left(1-\frac{u}{u_{e}}\right) \frac{\rho_{e}}{\rho} d z, \quad \tau_{w}(s)=\left(\mu \frac{\partial u}{\partial y}\right)_{y=0}=\frac{\rho_{w} \mu_{w}}{\rho_{0}} \frac{u_{e}}{\Delta^{* *}} \zeta ; \\
& \zeta(s)=\left[\frac{\partial\left(u / u_{e}\right)}{\partial\left(z / \Delta^{* *}\right)}\right]_{z=0}, \quad H=\frac{\Delta^{*}}{\Delta^{* *}}, \quad H_{1}=\frac{\Delta_{1}^{* *}}{\Delta^{* *}}, \quad N_{\sigma}=\frac{\rho_{0} \mu_{0}}{\rho_{w} \mu_{w}} \bar{N}, \quad \bar{N}=\frac{\sigma_{0} B_{m}^{2}}{\rho_{e}} .
\end{aligned}
$$

The characteristic function of the boundary layer $F_{m p}$ is:

$$
F_{m p}=2[\zeta-(2+H) f]+2 g H_{1}-2 \Lambda .
$$

The porosity parameter $\Lambda(s)$ is introduced with the expression:

$$
\Lambda(s)=-\frac{\mu_{0}}{\mu_{w}} \frac{v_{w} \Delta^{* *}}{v_{0}}=-\frac{V_{w} \Delta^{* *}}{v_{0}}=\Lambda_{1}(s) ; \quad V_{w}(s)=\frac{\mu_{0}}{\mu_{w}} v_{w}=\tilde{v}_{w}
$$

where the value $V_{w}(s)$ can be called the conditional transversal velocity at the inner edge of the boundary layer.

As with the incompressible fluid [3], the stream function $\psi(s, z)$ is divided into two parts, i.e. a new stream function $\psi^{*}(s, z)$ is introduced in the form of expression

$$
\psi(s, z)=\psi_{w}(s)+\psi^{*}(s, z), \quad \psi^{*}(s, 0)=0
$$

in which $\psi(s, 0)=\psi_{w}(s)$ denotes the stream function along the wall of the body within the fluid $(\mathrm{z}=0)$.

Applying (5), (6) and (11), the system (4), together with the boundary conditions trans- 
forms into:

$$
\begin{aligned}
& \frac{\partial \psi^{*}}{\partial z} \frac{\partial^{2} \psi^{*}}{\partial s \partial z}-\frac{\partial \psi^{*}}{\partial s} \frac{\partial^{2} \psi^{*}}{\partial z^{2}}-\frac{d \psi_{w}}{\frac{d s}{\underline{2}} \frac{\partial^{2} \psi^{*}}{\partial z^{2}}}=\frac{\rho_{e}}{\rho} u_{e} u_{e}^{\prime}+ \\
& +v_{0} \frac{\partial}{\partial z}\left(Q \frac{\partial^{2} \psi^{*}}{\partial z^{2}}\right)--\frac{\sigma_{0} B_{m}^{2}}{\rho u_{e}} \frac{\rho_{0} \mu_{0}}{\rho_{w} \mu_{w}}\left(u_{e}-\frac{\partial \psi^{*}}{\partial z}\right) \frac{\partial \psi^{*}}{\partial z}, \\
& \frac{\partial \psi^{*}}{\partial z} \frac{\partial h}{\partial s}-\frac{\partial \psi^{*}}{\partial s} \frac{\partial h}{\partial z}-\frac{d \psi_{w}}{\frac{\partial h}{\partial s} \frac{\partial h}{\partial z}}=-\frac{\rho_{e}}{\rho} u_{e} u_{e}^{\prime} \frac{\partial \psi^{*}}{\partial z}+v_{0} Q\left(\frac{\partial^{2} \psi^{*}}{\partial z^{2}}\right)^{2}+ \\
& +v_{0} \frac{\partial}{\partial z}\left(\frac{Q}{P r} \frac{\partial h}{\partial z}\right)+\frac{\sigma_{0} B_{m}^{2}}{\rho u_{e}} \frac{\rho_{0} \mu_{0}}{\rho_{w} \mu_{w}}\left(u_{e}-\frac{\partial \psi^{*}}{\partial z}\right)\left(\frac{\partial \psi^{*}}{\partial z}\right)^{2} ; \\
& \psi^{*}=0, \quad \frac{\partial \psi^{*}}{\partial z}=0, \quad h=h_{w} \quad \text { for }, z=0, \\
& \frac{\partial \psi^{*}}{\partial z} \rightarrow u_{e}(s), \quad h \rightarrow h_{e}(s) \quad \text { for } z \rightarrow \infty
\end{aligned}
$$

Both equations of the system (12) contain an additional (underlined) term on the left side of the equals sign. In these terms the derivative $d \psi_{w} / d s$ appears as

$$
\frac{d \psi_{w}(s)}{d s}=\frac{d \psi(s, 0)}{d s}=\left(\frac{\partial \psi}{\partial s}\right)_{z=0}=-\frac{\mu_{0}}{\mu_{w}} v_{w}=-V_{w}(s) .
$$

For the case of a nonporous wall of the body within the fluid (for which $v_{w}=0$ ) the underlined terms equal zero, which is logical, since these terms are the result of the porous contour of the body. The boundary conditions are the same as with the nonporous wall [8].

\section{Generalized boundary layer equations}

In order to obtain the generalized boundary layer equations, new transformations are introduced:

$$
\begin{aligned}
& s=s, \quad \eta(s, z)=\frac{u_{e}^{b / 2}}{K(s)} z, \quad \psi^{*}(s, z)=u_{e}^{1-b / 2} K(s) \Phi\left[\eta, \kappa,\left(f_{k}\right),\left(g_{k}\right),\left(\Lambda_{k}\right)\right], \\
& h(s, z)=h_{1} \cdot \bar{h}\left[\eta, \kappa,\left(f_{k}\right),\left(g_{k}\right),\left(\Lambda_{k}\right)\right], \quad, h_{e}+\frac{u_{e}^{2}}{2}=h_{1}=\text { const. } \\
& K(s)=\left(a v_{0} \int_{0}^{s} u_{e}^{b-1} d s\right)^{1 / 2} ; \quad a, b=\text { const. }
\end{aligned}
$$

The following notation is used: $\eta(s, z)$-newly introduced transversal variable, $\Phi$-newly introduced stream function, $\bar{h}$ - non-dimensional enthalpy; while $a$ and $b$ are constants that will be discussed later. 
Based on the expressions for the variable $\eta(s, z)$, certain important values and characteristics of the boundary layer (8) can be written as:

$$
\begin{aligned}
& u=u_{e} \frac{\partial \Phi}{\partial \eta}, \quad \Delta^{* *}(s)=\frac{K(s)}{u_{e}^{b / 2}} B(s), \quad B(s)=\int_{0}^{\infty} \frac{\partial \Phi}{\partial \eta}\left(1-\frac{\partial \Phi}{\partial \eta}\right) d \eta \\
& \frac{\Delta^{*}(s)}{\Delta^{* *}(s)}=H=\frac{A(s)}{B(s)}, \quad A(s)=\int_{0}^{\infty}\left(\frac{\rho_{e}}{\rho}-\frac{\partial \Phi}{\partial \eta}\right) d \eta, \quad \zeta=B\left(\frac{\partial^{2} \Phi}{\partial \eta^{2}}\right)_{\eta=0}, \quad A_{0}^{\infty} \\
& \frac{\Delta_{1}^{* *}(s)}{\Delta^{* *}(s)}=H_{1}=\frac{A_{1}(s)}{B(s)}, \quad A_{1}(s)=\int_{0}^{\infty} \frac{\partial \Phi}{\partial \eta}\left(1-\frac{\partial \Phi}{\partial \eta}\right) \frac{\rho_{e}}{\rho} d \eta, \quad \frac{f}{B^{2}}=\frac{a u_{e}^{\prime}}{u_{e}^{b}} \int_{0}^{s} u_{e}^{b-1} d s .
\end{aligned}
$$

Here, it is assumed that the already defined values $A, B$ and $A_{1}$ are continual functions of the variable $s$.

With the non-dimensional functions $\Phi$ and $\bar{h}$, in the general similarity transformations (14), we introduced a local parameter of the ionized gas compressibility $\kappa=f_{0}$, a set of parameters of the form $f_{k}$ of Loitsianskii type [3], a set of magnetic parameters $g_{k}$ and a set of porosity parameters $\Lambda_{k}[8]$. These sets are determined by the expressions:

$$
\begin{aligned}
& \kappa=f_{0}(s)=\frac{u_{e}^{2}}{2 h_{1}}, \quad f_{k}(s)=u_{e}^{k-1} u_{e}^{(k)} Z^{* * k}, \quad g_{k}(s)=u_{e}^{k-1}, \quad N_{\sigma}^{(k-1)} Z^{* * k}, \\
& \Lambda_{k}(s)=-u_{e}^{k-1}\left(\frac{V_{w}}{\sqrt{v_{0}}}\right)^{(k-1)} Z^{* * k-1 / 2}, \quad(k=1,2,3, \ldots)
\end{aligned}
$$

and they present independent variables instead of the longitudinal variable $s$.

Just like with incompressible fluid, the parameters of the sets (16) satisfy the corresponding simple recurrent differential equations of the form:

$$
\begin{aligned}
& \frac{u_{e}}{u_{e}^{\prime}} f_{1} \frac{d \kappa}{d s}=2 \kappa f_{1}=\theta_{0}, \\
& \frac{u_{e}}{u_{e}^{\prime}} f_{1} \frac{d f_{k}}{d s}=\left[(k-1) f_{1}+k F_{m p}\right] f_{k}+f_{k+1}=\theta_{k}, \\
& \frac{u_{e}}{u_{e}^{\prime}} f_{1} \frac{d g_{k}}{d s}=\left[(k-1) f_{1}+k F_{m p}\right] g_{k}+g_{k+1}=\gamma_{k}, \\
& \frac{u_{e}}{u_{e}^{\prime}} f_{1} \frac{d \Lambda_{k}}{d s}=\left\{(k-1) f_{1}+[(2 k-1) / 2] F_{m p}\right\} \Lambda_{k}+\Lambda_{k+1}=\chi_{k} \cdot(k=1,2,3, \ldots)
\end{aligned}
$$

Applying the similarity transformations (14) and (16) to the equation system (12) the generalized boundary layer equation system is obtained. Together with the transformed boundary 
condition the system takes the following form:

$$
\begin{aligned}
& \frac{\partial}{\partial \eta}\left(Q \frac{\partial^{2} \Phi}{\partial \eta^{2}}\right)+\frac{a B^{2}+(2-b) f_{1}}{2 B^{2}} \Phi \frac{\partial^{2} \Phi}{\partial \eta^{2}}+\frac{f_{1}}{B^{2}}\left[\frac{\rho_{e}}{\rho}-\left(\frac{\partial \Phi}{\partial \eta}\right)^{2}\right]-\frac{g_{1}}{B^{2}} \frac{\rho_{e}}{\rho}\left(1-\frac{\partial \Phi}{\partial \eta}\right) \frac{\partial \Phi}{\partial \eta}+ \\
& +\underline{\underline{\underline{\Lambda_{1}}} \frac{\partial^{2} \Phi}{\partial \eta^{2}}}=\frac{1}{B^{2}}\left[\sum_{k=0}^{\infty} \theta_{k}\left(\frac{\partial \Phi}{\partial \eta} \frac{\partial^{2} \Phi}{\partial \eta \partial f_{k}}-\frac{\partial \Phi}{\partial f_{k}} \frac{\partial^{2} \Phi}{\partial \eta^{2}}\right)+\sum_{k=1}^{\infty} \gamma_{k}\left(\frac{\partial \Phi}{\partial \eta} \frac{\partial^{2} \Phi}{\partial \eta \partial g_{k}}-\frac{\partial \Phi}{\partial g_{k}} \frac{\partial^{2} \Phi}{\partial \eta^{2}}\right)+\right. \\
& \left.+\sum_{k=1}^{\infty} \chi_{k}\left(\frac{\partial \Phi}{\partial \eta} \frac{\partial^{2} \Phi}{\partial \eta \partial \Lambda_{k}}-\frac{\partial \Phi}{\partial \Lambda_{k}} \frac{\partial^{2} \Phi}{\partial \eta^{2}}\right)\right] \\
& \frac{\partial}{\partial \eta}\left(\frac{Q}{\operatorname{Pr}} \frac{\partial \bar{h}}{\partial \eta}\right)+\frac{a B^{2}+(2-b) f_{1}}{2 B^{2}} \Phi \frac{\partial \bar{h}}{\partial \eta}-\frac{2 \kappa f_{1}}{B^{2}} \frac{\rho_{e}}{\rho} \frac{\partial \Phi}{\partial \eta}+2 \kappa Q\left(\frac{\partial^{2} \Phi}{\partial \eta^{2}}\right)^{2}+ \\
& +\frac{2 \kappa g_{1}}{B^{2}} \frac{\rho_{e}}{\rho}\left(1-\frac{\partial \Phi}{\partial \eta}\right)\left(\frac{\partial \Phi}{\partial \eta}\right)^{2}+\underline{\underline{\frac{\Lambda_{1}}{B} \frac{\partial \bar{h}}{\partial \eta}}}=\frac{1}{B^{2}}\left[\sum_{k=0}^{\infty} \theta_{k}\left(\frac{\partial \Phi}{\partial \eta} \frac{\partial \bar{h}}{\partial f_{k}}-\frac{\partial \Phi}{\partial f_{k}} \frac{\partial \bar{h}}{\partial \eta}\right)+\right. \\
& \left.+\sum_{k=1}^{\infty} \gamma_{k}\left(\frac{\partial \Phi}{\partial \eta} \frac{\partial \bar{h}}{\partial g_{k}}-\frac{\partial \Phi}{\partial g_{k}} \frac{\partial \bar{h}}{\partial \eta}\right)+\sum_{k=1}^{\infty} \chi_{k}\left(\frac{\partial \Phi}{\partial \eta} \frac{\partial \bar{h}}{\partial \Lambda_{k}}-\frac{\partial \Phi}{\partial \Lambda_{k}}, \frac{\partial \bar{h}}{\partial \eta}\right)\right] ; \\
& \Phi=\frac{\partial \Phi}{\partial \eta}=0, \quad \bar{h}=\bar{h}_{w}=\text { const. } \quad \text { for } \eta=0 \\
& \frac{\partial \Phi}{\partial \eta} \rightarrow 1, \quad \bar{h} \rightarrow \bar{h}_{e}=1-\kappa \quad \text { for } \eta \rightarrow \infty
\end{aligned}
$$

Distribution of the outer velocity $u_{e}(s)$ appears neither in the equations of the obtained system nor in the corresponding boundary conditions. Therefore, the equation system (18) represents a general mathematical model for the case of the ionized gas flow in the boundary layer adjacent to a porous wall of the body within the fluid when the law on electroconductivity variation is given with the expression (3).

\section{Numerical solution}

Numerical solution of the system (18) is possible only in the $n$ - parametric approximation. If assumed that all the parameters equal zero, starting from the second one, i.e., if $f_{k}=0, \quad g_{k}=0$ and $\Lambda_{k}=0$ when $k \geq 2$, the obtained equation system is significantly simplified. The system (21) comes down to the system of partial differential equations with five independent variables: $\eta, \kappa, f_{1}, g_{1}, \Lambda_{1}$, ; and it represents a four-parametric approximation. In the boundary layer theory, it is common $[3,9]$ to neglect the first derivatives per some of the mentioned parameters, i.e., to perform the so-called localization. Here, the localization is justified due to difficulties of mathematical nature. Therefore, the equation system for numerical solution in the four-parametric three times localized approximation $\left(\partial / \partial \kappa=0, \partial / \partial g_{1}=0, \partial / \partial \Lambda_{1}=0\right)$ together with the corresponding boundary conditions 
goes as:

$$
\begin{gathered}
\frac{\partial}{\partial \eta}\left(Q \frac{\partial^{2} \Phi}{\partial \eta^{2}}\right)+\frac{a B^{2}+(2-b) f}{2, B^{2}} \Phi \frac{\partial^{2} \Phi}{\partial \eta^{2}}+\frac{f}{B^{2}}\left[\frac{\rho_{e}}{\rho}-\left(\frac{\partial \Phi}{\partial \eta}\right)^{2}\right]- \\
-\frac{g}{B^{2}} \frac{\rho_{e}}{\rho}\left(1-\frac{\partial \Phi}{\partial \eta}\right) \frac{\partial \Phi}{\partial \eta}+\frac{\Lambda}{\underline{B} \frac{\partial^{2} \Phi}{\partial \eta^{2}}}=\frac{F_{m p} f}{B^{2}}\left(\frac{\partial \Phi}{\partial \eta} \frac{\partial^{2} \Phi}{\partial \eta \partial f}-\frac{\partial \Phi}{\partial f} \frac{\partial^{2} \Phi}{\partial \eta^{2}}\right), \\
\frac{\partial}{\partial \eta}\left(\frac{Q}{\operatorname{Pr}} \frac{\partial \bar{h}}{\partial \eta}\right)+\frac{a B^{2}+(2-b) f}{2 B^{2}} \Phi \frac{\partial \bar{h}}{\partial \eta}-\frac{2 \kappa f}{B^{2}} \frac{\rho_{e}}{\rho} \frac{\partial \Phi}{\partial \eta}+2 \kappa Q\left(\frac{\partial^{2} \Phi}{\partial \eta^{2}}\right)^{2}+ \\
+\frac{2 \kappa g}{B^{2}} \frac{\rho_{e}}{\rho}\left(1-\frac{\partial \Phi}{\partial \eta}\right)\left(\frac{\partial \Phi}{\partial \eta}\right)^{2}+\frac{\Lambda}{B} \frac{\partial \bar{h}}{\partial \eta}=\frac{F_{m p} f}{B^{2}}\left(\frac{\partial \Phi}{\partial \eta} \frac{\partial \bar{h}}{\partial f}-\frac{\partial \Phi}{\partial f} \frac{\partial \bar{h}}{\partial \eta}\right) ; \\
\Phi=\frac{\partial \Phi}{\partial \eta}=0, \quad \quad \quad \quad h=\bar{h}_{w}=\text { const. } \quad \text { for } \eta=0, \\
\frac{\partial \Phi}{\partial \eta} \rightarrow 1, \quad \bar{h} \rightarrow \bar{h}_{e}=1-\kappa \quad \text { for } \eta \rightarrow \infty .
\end{gathered}
$$

Note that in the system (19), the index 1 is left out from some (first) parameters. Both of the equations of the system (19) contain the underlined term that characterizes the porous wall of the body within the fluid.

Therefore, the equation system (19) represents a general mathematical model of the studied problem. For numerical integration of the obtained system of differential partial equations of the third order, it is necessary first to decrease the order of the dynamic equation. By the usual transformation $[8,9]$

$$
u / u_{e}=\partial \Phi / \partial \eta=\varphi
$$

the order of the dynamic equation is decreased, hence the corresponding equation system is:

$$
\begin{aligned}
& \frac{\partial}{\partial \eta}\left(Q \frac{\partial \varphi}{\partial \eta}\right)+\frac{a B^{2}+(2-b) f}{2 B^{2}} \Phi \frac{\partial \varphi}{\partial \eta}+\frac{f}{B^{2}}\left[\frac{\rho_{e}}{\rho}-\varphi^{2}\right]-\frac{g}{B^{2}} \frac{\rho_{e}}{\rho}(1-\varphi) \varphi+ \\
& +\frac{\Lambda}{B} \frac{\partial \varphi}{\partial \eta}=\frac{F_{m p} f}{B^{2}}\left(\varphi \frac{\partial \varphi}{\partial f}-\frac{\partial \Phi}{\partial f} \frac{\partial \varphi}{\partial \eta}\right), \\
& \frac{\partial}{\partial \eta}\left(\frac{Q}{\operatorname{Pr}} \frac{\partial \bar{h}}{\partial \eta}\right)+\frac{a B^{2}+(2-b) f}{2 B^{2}} \Phi \frac{\partial \bar{h}}{\partial \eta}-\frac{2 \kappa f}{B^{2}} \frac{\rho_{e}}{\rho} \varphi+2 \kappa Q\left(\frac{\partial \varphi}{\partial \eta}\right)^{2}+ \\
& +\frac{2 \kappa g}{B^{2}} \frac{\rho_{e}}{\rho}(1-\varphi) \varphi^{2}+\frac{\Lambda}{\frac{\partial}{\partial \eta}} \frac{F_{m p}}{\partial \eta}\left(\varphi \frac{\partial \bar{h}}{\partial f}-\frac{\partial \Phi}{\partial f} \frac{\partial \bar{h}}{\partial \eta}\right) ; \\
& \Phi=\varphi=0, \quad \bar{h}=\bar{h}_{w}=\text { const. } \quad \text { for }, \eta=0 \\
& \varphi \rightarrow 1, \quad \bar{h} \rightarrow \bar{h}_{e}=1-\kappa, \quad \text { for } \text { eta } \rightarrow \infty .
\end{aligned}
$$


A concrete numerical solution of the system of nonlinear and conjugated differential partial equations (21) is done by finite differences method, i.e., ?passage method? or TDA method. Based on the planar integration grid [9], derivatives of the functions $\varphi, \Phi$ and $\bar{h}$ are substituted with finite differences ratios. For the nondimensional function $Q$ [8] and for the density ratio $\rho_{e} / \rho$ that appear in the equations (21), approximate formulas are used in the forms of:

$$
Q=Q(\bar{h}) \approx\left(\bar{h}_{w} / \bar{h}\right)^{1 / 3}, \quad \rho_{e} / \rho \approx \bar{h} /(1-\kappa) .
$$

These expressions are more appropriate for the dissociated gas flow, and in the paper [4] they are determined based on the tables of the thermodynamic functions for the air. For the numerical solution of the system (21), a program is written in FORTRAN programming language. All the calculations in this paper are done for the concrete values of the constants $a$ and $b$ when $a=0.4408 ; b=5.7140$ that according to [9] represent the optimal values. Prandtl number is taken to be constant - $\operatorname{Pr}=0.712$.

Since the equation system (21) is localized per the compressibility, porosity and magnetic parameter; all these parameters have the role of simple parameters. That is why the system (21) is solved by the usual procedure [9], starting from the value $f=0.00$ (flat plate), for the given values of the parameters $\kappa, g$ and $\Lambda$.

\section{Results and conclusions}

Numerical solutions of the system (21) are obtained in the form of tables defined by the written program. Each of the tables represents the solution of the equation system in the corresponding cross-section of the boundary layer for the given values of the input parameters $(\kappa, g, \Lambda)$.Only some of the most important results in the form of diagrams are presented in this paper.

Diagrams for the non-dimensional velocity $u / u_{e}$ (Fig. 1), non-dimensional enthalpy $\bar{h}$ (Fig. 2), quantity $A$ (Fig. 3) and $B$ (Fig. 4), non-dimensional friction function $\zeta$ (Fig. 5 and Fig. 6), and the diagram for characteristic function $F_{m p}$ (Fig. 7) are shown here. Diagrams for distribution of the non-dimensional velocity (Fig. 8), non-dimensional function $\zeta$ (Fig. 9 ) and the diagram of the characteristic function $F_{m p}$ (Fig. 10) are also given for different values of the porosity parameter $\Lambda$.

Based on the given and other diagrams [8] the following important conclusions have been drawn:

- The solution profiles of the boundary layer equations are the same as with other problems of compressible fluid flow.

- The non-dimensional velocity $u / u_{e}$ in each of the cross-sections of the boundary layer (different $f$ ) very quickly converges towards unity (Fig. 1). 
- The magnetic parameter $g$ has a significant influence on the boundary layer quantities $A, B, \zeta$ and $F_{m p}$ (Fig. 3-7).

- With increase in values of the parameter $g$, the boundary separation point moves down the current (Fig. 5 and Fig. 6).

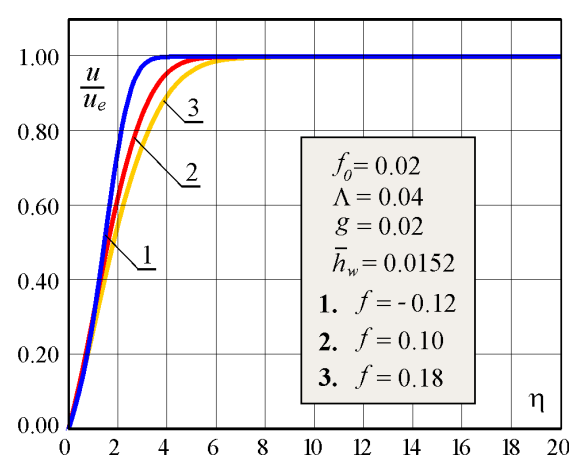

Fig. 1: Diagram of the non-dimensional velocity $u / u_{e}$

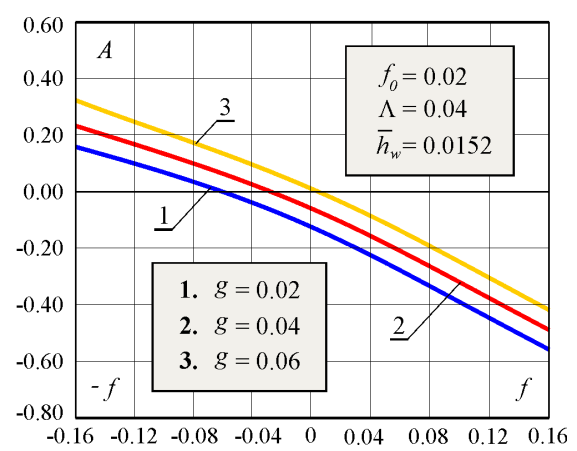

Fig. 3: Graphic of the characteristic $A$ of the boundary layer

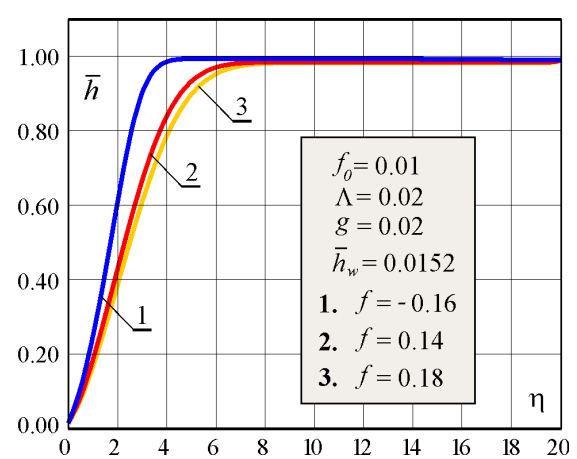

Fig. 2: Diagram of the non-dimensional enthalpy $\bar{h}$

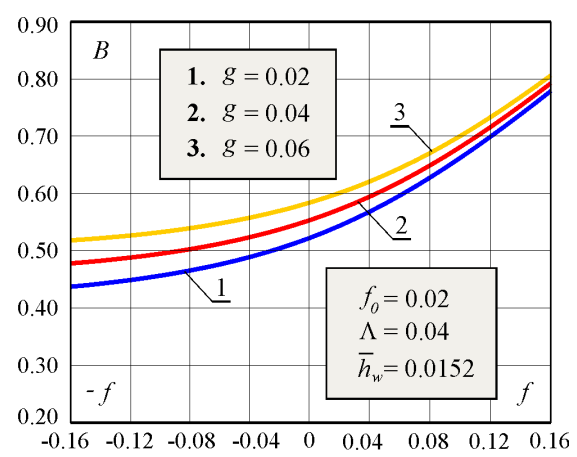

Fig. 4: Graphic of the characteristic $B$ of the boundary layer

- The ionized gas compressibility parameter $f_{0}$ does not have a significant influence on the non-dimensional friction function $\zeta$. This influence is very small when the value of the form parameter is $f=0$ (flat plate), but it increases closer to the boundary layer separation point (Fig. 5 and Fig. 6). For example, at the cross-section of the boundary layer for which $f=0, g=0.04$ and $\Lambda=0.04$, when the parameter $f_{0}$ increases from 0.02 to 0.20 , the non-dimensional friction function increases from 0.338 to 0.346 , i.e., it increases 1.024 times. However, within the diffusor region of the boundary 


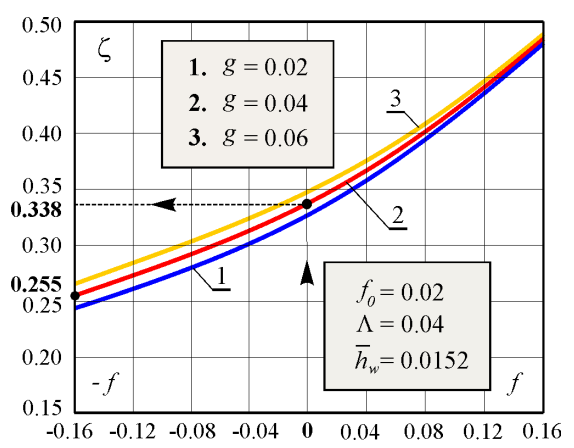

Fig. 5: Distribution of the non-dimensional friction function $\zeta\left(f_{0}=0.02\right)$

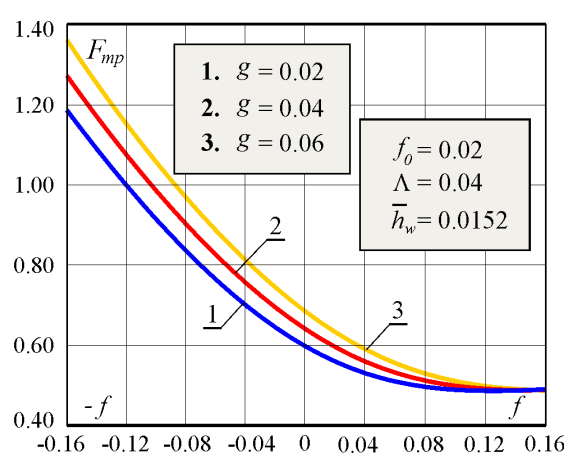

Fig. 7: Characteristic function of the boundary layer $F_{m p}$

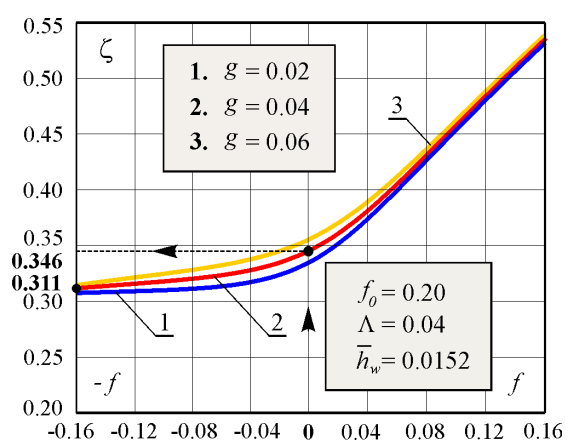

Fig. 6: Distribution of the non-dimensional friction function $\zeta\left(f_{0}=0.20\right)$

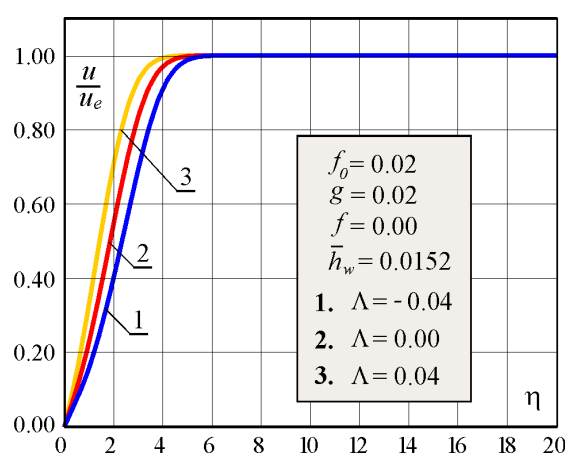

Fig. 8: Diagrams of the non-dimensional velocity $u / u_{e}$ for different values of $\Lambda(f=$ $0.00)$

layer when $f=-0.16$ and the values of other input parameters remain unchanged, the influence of the compressibility parameter is slightly increased. In that case the function $\zeta$ increases from 0.255 to 0.311 , i.e., it increases 1.220 times.

- The porosity parameter $\Lambda$ has a relatively small influence of on the non-dimensional velocities profiles $u / u_{e}$ (Fig. 8). Regardless of the fact whether the ionized gas is injected into the main current or ejected, at different cross-sections of the boundary layer (e.g. $f=0$ and $f=0.08$ ), the non-dimensional velocity converges towards unity very fast.

- Figure 9 shows that the porosity parameter $\Lambda$ has a great influence on the nondimensional friction function $\zeta$. When values of other input parameters remain unchanged, a decrease in the value of this parameter delays the separation of the boundary layer. 


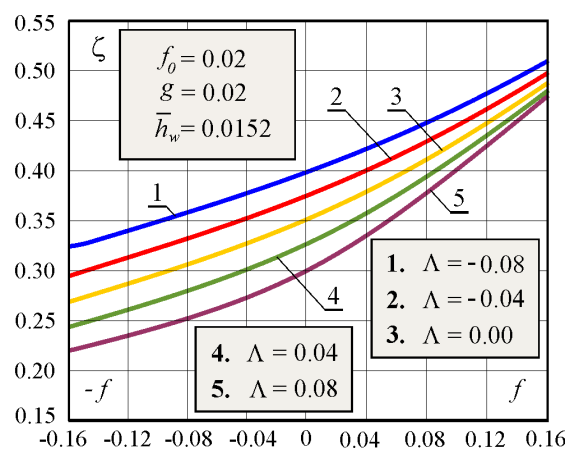

Fig. 9: Distribution of the non-dimensional friction function $\zeta$ for different values of $\Lambda$

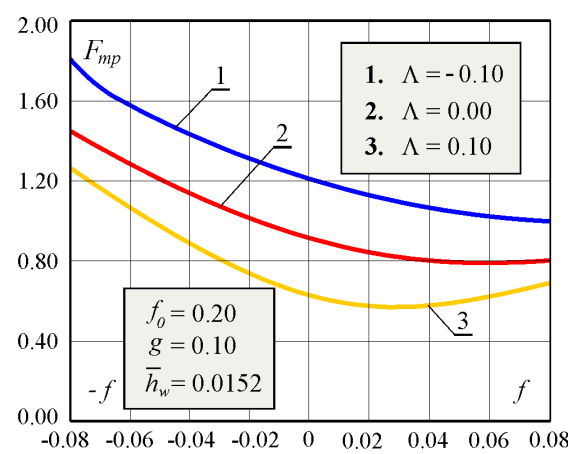

Fig. 10: Distribution of the characteristic function $F_{m p}$ for different values of $\Lambda$

- The porosity parameter $\Lambda$ has a significant influence on the characteristic boundary layer function $F_{m p}$ on a porous wall (Fig. 10).

These conclusions are almost identical to the conclusions drawn for ionized gas flow in the boundary layer adjacent to either a porous or nonporous body contour regardless of the gas electro-conductivity variation law.

The given conclusions are also compliant with the conclusions drawn for different compressible fluid flow problems. From the aspect of physics, the conducted investigations present a significant contribution to the ionized gas boundary layer theory.

\section{Acknowledgment}

The research was supported by the Ministry of Education and Science of the Republic of Serbia Grant ON174014.

\section{References}

[1] W. H. DorRance, Viscous Hypersonic Flow, Theory of Reacting and Hypersonic Boundary Layers (in Russian), Mir, Moscow, 1966.

[2] L. G. Loitsians KII, Laminar Boundary Layer (in German), Akademie-Verlag, Berlin, 1967.

[3] L. G. LoitsianskiI, Mechanics of Liquids and Gases, Begell House Publishers, New York, 1995.

[4] N. V. KRIVTSOVA, Laminar Boundary Layer in an Equilibrium Dissociated Gas for Arbitrary External Velocity Distribution, Fluid Dynamics Vol. 1, 5 (1966), 73-76.

[5] V. Saljnikov, Z. Boričić, D. Nikodijević, Parametric Method in Unsteady MHD Boundary Layer Theory of Fluid with Variable Electroconductivity, Facta Universitatis, Series: Mechanics, Automatic Control and Robotics Vol. 2, 7/2 (1997), 331-340. 
[6] Z. BoričIĆ, D. NiKodiJević, B. OBRović, Unsteady flow of the fluid, whose electroconductivity is a function of the longitudinal velocity gradient, in MHD boundary layer on a body (in Serbian), XX Yugoslav Congress of Theoretical and Applied Mechanics, Kragujevac, 1993, pp. 136-139.

[7] B. Obrović, S. SAVić, Ionized Gas Boundary Layer on a Porous Wall of the Body within the Electroconductive Fluid, Theoret. Appl. Mech. Vol. 31, 1 (2004), 47-71.

[8] S. SAVIĆ, Solution of the Problem of the Ionized Gas Flow in the Boundary Layer in Case of a Nonporous and a Porous Contour of the Body within the Fluid (in Serbian), Ph. D. Thesis, University of Kragujevac, Faculty of Mechanical Engineering, Kragujevac, 2006.

[9] V. Saljnikov, U. Dallmann, Verallgemeinerte Ähnlichkeitslösungen für dreidimensionale, laminare, stationäre, kompressible Grenzschichtströmungen an schiebenden profilierten Zylindern, Institut für Theoretische Strömungsmechanik, Göttingen, 1989.

[10] B. Obrović, D. Nikodijević, S. SAVić, Boundary Layer of the Dissociated Gas Flow Over a Porous Wall Under the Conditions of Equilibrium Dissociation, Theoret. Appl. Mech. Vol. 32, 2 (2005), 167-192. 